\title{
Radiation-induced autophagy promotes esophageal squamous cell carcinoma cell survival via the LKB1 pathway
}

\author{
CHI LU ${ }^{1,2}$ and CONGHUA XIE ${ }^{1,2}$ \\ ${ }^{1}$ Department of Radiation and Medical Oncology, Zhongnan Hospital, Wuhan University, Wuhan, Hubei 430014; \\ ${ }^{2}$ Hubei Key Laboratory of Tumor Biological Behaviors, Wuhan University, Wuhan, Hubei 430014, P.R. China
}

Received December 17, 2015; Accepted January 27, 2016

DOI: $10.3892 /$ or.2016.4753

\begin{abstract}
Radiotherapy is an important treatment modality for esophageal cancer; however, the clinical efficacy of radiotherapy is limited by tumor radioresistance. In the present study, we explored the hypothesis that radiation induces tumor cell autophagy as a cytoprotective adaptive response, which depends on liver kinase B1 (LKB1) also known as serine/ threonine kinase 11 (STK11). Radiation-induced Eca-109 cell autophagy was found to be dependent on signaling through the LKB1 pathway, and autophagy inhibitors that disrupted radiation-induced Eca-109 cell autophagy increased cell cycle arrest and cell death in vitro. Inhibition of autophagy also reduced the clonogenic survival of the Eca-109 cells. When treated with radiation alone, human esophageal carcinoma xenografts showed increased LC3B and p-LKB1 expression, which was decreased by the autophagy inhibitor chloroquine. In vivo inhibition of autophagy disrupted tumor growth and increased tumor apoptosis when combined with $6 \mathrm{~Gy}$ of ionizing radiation. In summary, our findings elucidate a novel mechanism of resistance to radiotherapy in which radiationinduced autophagy, via the LKB1 pathway, promotes tumor cell survival. This indicates that inhibition of autophagy can serve as an adjuvant treatment to improve the curative effect of radiotherapy.
\end{abstract}

\section{Introduction}

Esophageal carcinoma (EC) is the eighth most common malignancy and the sixth leading cause of cancer-related death worldwide (1). In China, EC is the fourth leading cause of cancer-associated death (2). EC remains an aggressive disease with a high mortality rate. Despite major progress in clinical diagnostics and therapy, EC represents a tumor entity with a limited prognosis. The overall survival of EC patients remains

Correspondence to: Dr Conghua Xie, Department of Radiation and Medical Oncology, Zhongnan Hospital, Wuhan University, Wuhan, Hubei 430014, P.R. China

E-mail: chxie_65@whu.edu.cn

Key words: autophagy, esophageal cancer, LKB1, radiation poor, and the 5-year survival rate is $17 \%$ (3-5). In multimodal therapy concepts of locally advanced EC, radiotherapy is one of the well-established therapeutic modalities for cancer treatment (6). However, radioresistance is a major challenge during the treatment of EC. A better understanding of the mechanisms of radioresistance may identify strategies through which to address this challenge.

Autophagy is an evolutionarily conserved process designed for the degradation and turnover of long-lived proteins and dysfunctional organelles. Activation of autophagic pathways can serve as a survival and adaptive mechanism, providing metabolic support in times of cellular stress, such as exposure to radiation, limiting the efficacy of radiotherapy (6). However, few studies have shown that autophagy is a protective mechanism against radiation damage for tumor cells (7). Autophagy protects the cells against radiation damage by supplying the products of catabolism to repair cells and by eliminating damaged cells and protein aggregates, but whether the response is actually cytoprotective and the identity of the pathways involved remain undetermined. Sapkota et al reported that the tumor-suppressor protein LKB1 (serinethreonine kinase liver kinase B1) is induced by activated ATM after radiation (8). Furthermore, AMPK and LKB1, which are signaling molecules, stimulate autophagic activity via p27 or mTOR signaling (9). Therefore, we hypothesized that radiation promotes autophagy as a cytoprotective adaptive mechanism via the LKB1 pathway.

Here, we report that autophagy was induced during exposure to radiation and promoted esophageal squamous cell carcinoma cell survival. We then investigated the role of the LKB1 pathway in activating autophagy. We demonstrated the effects of this novel mechanism in vitro and in vivo and showed that pharmacological or genetic autophagy inhibition contributed to EC cell radiosensitization.

\section{Materials and methods}

Cells and reagents. The esophageal carcinoma cell line Eca-109 was obtained from the China Center For Type Culture Collection. A total of $1 \times 10^{5}$ cells/well were plated in 6 -well plates in RPMI-1640 medium containing $10 \%$ fetal calf serum (FCS) and were incubated in a humidified atmosphere of $95 \%$ air and $5 \% \mathrm{CO}_{2}$ at $37^{\circ} \mathrm{C}$. The primary antibodies were against LC3B, phospho-AMPK $\alpha$ (Thr 172), LKB1 (all 
from Cell Signaling Technology), and GAPDH (Abcam). The phospho(p)-LKB1 (Thr 366) antibody was produced by Sangon Biotech (Shanghai, China) against the peptide KIEDGIIYTQDFTVPGK, in which the underlined residue is a phosphothreonine. 3-Methyladenine (3-MA) and chloroquine diphosphate (CQ) were purchased from Sigma-Aldrich.

Western blotting. Cell lysates were centrifuged at 12,000 rpm for $5 \mathrm{~min}$ at $4^{\circ} \mathrm{C}$. The supernatants were collected, and $30 \mu \mathrm{g}$ of protein was electrophoresed on 12\% SDS-PAGE gels, transferred to PVDF membranes, and incubated with the primary antibody at $4^{\circ} \mathrm{C}$ overnight. Goat anti-rabbit IgG secondary antibody conjugated to horseradish peroxidase and a chemiluminescence system (Thermo Fisher Scientific) were used for detection. The band intensities were determined by densitometry using ImageJ software.

GFP-LC3 redistribution evaluation. Eca-109 cells were transfected with PBABEpuro GFP-LC3 plasmid (Addgene, Cambridge, MA, USA) using the MACSfectin ${ }^{\mathrm{TM}}$ reagent (Miltenyi, Bergisch Gladbach, Germany). Quantification of green dots was performed using the particle analysis plugin of ImageJ (NIH). A cell was considered to be GFP-LC3-positive when it had 10 or more green dots per cell, and a total of 100 cells were counted per treatment.

Viability assay. Cells were seeded at a density of $5 \times 10^{4} / \mathrm{ml}$ in 96-well plates. After $24 \mathrm{~h}$, the cells were treated with a singledose of radiation $(0,2,4$, or $6 \mathrm{~Gy})$, and CQ was added $1 \mathrm{~h}$ before irradiation in the experimental study groups. At $24 \mathrm{~h}$ after irradiation, $20 \mu \mathrm{l}$ of a $5 \mathrm{mg} / \mathrm{ml}$ MTT solution was added to each well, and the plates were incubated at $37^{\circ} \mathrm{C}$ for $4 \mathrm{~h}$. The medium was removed, and $150 \mu 1$ of DMSO per well was added. The absorbance was measured at $490 \mathrm{~nm}$ with a Enspire Multimode reader. The percentage of cell survival was calculated relative to the control cells, which was set to $100 \%$.

Hoechst 33342 staining. Eca-109 cells were plated in 6-well plates and fixed with $4 \%$ paraformaldehyde for $10 \mathrm{~min}$ at $25^{\circ} \mathrm{C}$. Then, the cells were washed twice with PBS. Hoechst 33342 staining ( $1 \mathrm{mM}$; Sigma-Aldrich) solution was added to the plate and incubated for $15 \mathrm{~min}$ in the dark at $37^{\circ} \mathrm{C}$. The staining solution was then discarded, and the cells were washed twice with PBS. Stained cells were observed by fluorescence microscopy (Olympus).

Flow cytometric analysis. Cells were collected $24 \mathrm{~h}$ after radiation. For cell cycle distribution analysis, the cells were stained with propidium iodide (PI) $(50 \mu \mathrm{g} / \mathrm{ml})$ solution. For apoptosis detection, the cells were stained with the Annexin V-FITC apoptosis detection kit (Abcam). Stained cells were detected by flow cytometry (BD Biosystems, USA), and data were analyzed with the ModFit LT v2.0 and CellQuest software programs.

Clonogenic assay. Cells were treated with single-dose irradiation $(0,1,2,4$, or $6 \mathrm{~Gy})$, and CQ was added $1 \mathrm{~h}$ before irradiation in the experimental study groups. Irradiation was performed at $37^{\circ} \mathrm{C}$, and the dose rate was $0.4 \mathrm{~Gy} / \mathrm{min}$. At $24 \mathrm{~h}$ after irradiation, varying cell numbers were seeded in tripli- cate in $60-\mathrm{mm}$ culture dishes with $5 \mathrm{ml}$ of regular cell culture medium and incubated for 7-14 days. The cells were fixed with ethanol and then stained with $0.5 \%$ crystal violet. Colonies that contained $\geq 50$ cells were counted.

siRNA transfection. A total of $1 \times 10^{6}$ cells/well in 12 -well plates were transfected overnight using MACSfection reagent (Miltenyi) with LKB1 siRNA, AMPK $\alpha$ siRNA (both from Santa Cruz Biotechnology) or a negative control. After culture with fresh medium for $24 \mathrm{~h}$, knockdown was confirmed by western blotting.

$R N A$ isolation and conventional reverse transcription-PCR. Total cellular RNA from $5 \times 10^{5}$ cells was prepared with TRIzol reagent (Invitrogen). A total of $1 \mu \mathrm{g}$ of RNA per sample was converted to cDNA with the Qiagen OneStep RT-PCR kit (Qiagen) using a C1000 Touch Thermal Cycler (Bio-Rad). The cDNA synthesis reactions were pre-incubated at $94^{\circ} \mathrm{C}$ and then amplified over 30 cycles consisting of a 30 -sec melting step $\left(94^{\circ} \mathrm{C}\right)$, a 15 -sec annealing step at $58^{\circ} \mathrm{C}$, and a 25 -sec extension step $\left(72^{\circ} \mathrm{C}\right)$.

Real-time RT-PCR. We reverse-transcribed $1 \mu \mathrm{g}$ of RNA with Reverse Transcriptase M-MLV (Takara). PCR reactions were performed in triplicate with the SYBR Green Master Mix (Toyobo). Reactions were pre-incubated at $95^{\circ} \mathrm{C}$ and then amplified over 40 cycles consisting of a 30 -sec melting step $\left(94^{\circ} \mathrm{C}\right.$ ), a $15-\mathrm{sec}$ annealing step at $58^{\circ} \mathrm{C}$, and a 25 -sec extension step $\left(72^{\circ} \mathrm{C}\right)$. Amplification was measured using a CFX96 Real-Time PCR detection system (Bio-Rad). The primers included LC3B forward, GGT AAA CGG GCT GTG TGA GA and reverse, AAG GCA GAA GGG AGT GTG TC; and LKB1 forward, ACT GAG GAG GTT ACG GCA VA and reverse, TGG TGA TGT TGT AGA GGG TGA.

Xenografts. Animal procedures were approved by the University Committee on the Use and Care of Animals of the Huazhong University of Science and Technology. A total of $1 \times 10^{6}$ Eca-109 cells were injected subcutaneously into the right posterior limb of 6- to 8-week old male Balb/c nu/nu mice. After the tumors were established (mean subcutaneous volume, $50 \mathrm{~mm}^{3}$ ), the mice were randomly divided into four groups. Groups 1 and 2 were injected intraperitoneally with PBS or CQ $(50 \mathrm{mg} / \mathrm{kg})$, respectively. Group 3 was administered a single dose of localized irradiation (6 Gy), and group 4 was irradiated and then administered an intraperitoneal injection of CQ. All of the experiments used five mice per group. Tumors were measured with calipers twice weekly. The tumor volume was the length $\mathrm{x}$ (width) $)^{2} / 2$, and fold-growth was relative to treatment day one. Tumor tissues were removed on day 27.

Immunohistochemistry. Slides were stained with primary antibody overnight at $4^{\circ} \mathrm{C}$ followed by HRP-conjugated secondary antibodies (1:250) at room temperature for $30 \mathrm{~min}$. TUNEL staining was performed according to the manufacturer's instructions. The One Step TUNEL apoptosis assay kit was obtained from the Beyotime Institute of Biotechnology (China). Afterwards, the slides were observed under an Olympus BX51 research microscope and images were obtained. The cells with green fluorescence were defined as apoptotic cells. 

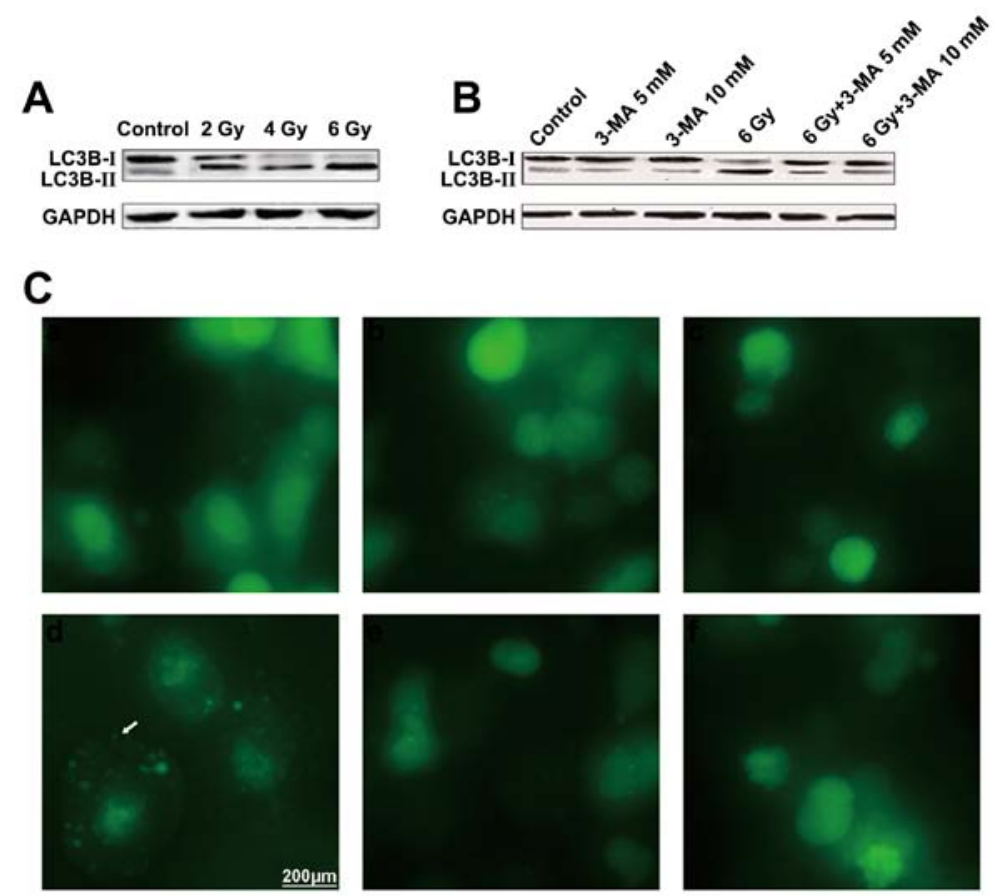

D

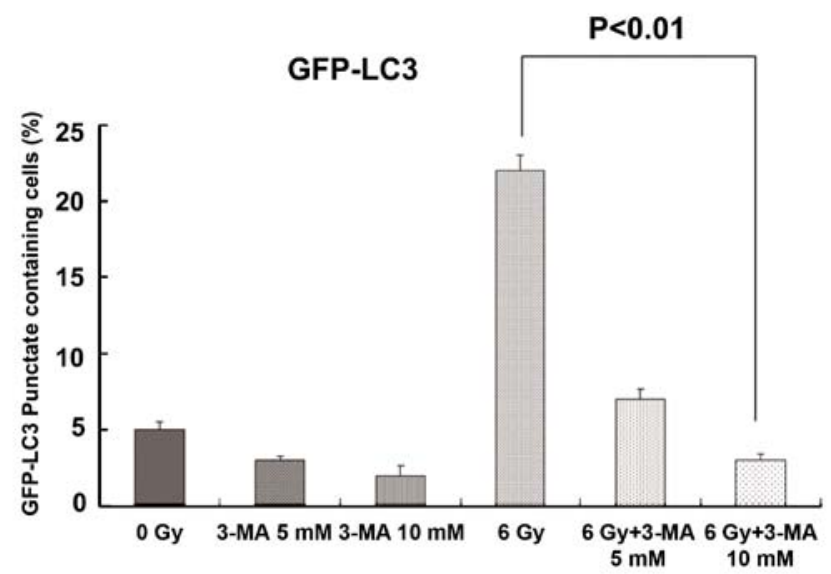

Figure 1. Autophagy inhibitors block the radiation-induced expression of autophagy mediators in Eca-109 cells. (A) Western blot analysis for the detection of the conversion of LC3B-I to LC3B-II in Eca-109 cells $24 \mathrm{~h}$ after exposure to different radiation doses. (B) Western blot analysis for the detection of LC3B levels in Eca-109 cells $24 \mathrm{~h}$ after exposure to single or combined treatments. Combined therapy with ionizing radiation of $6 \mathrm{~Gy}$ and 3-MA (5 or $10 \mathrm{mM}$ ) inhibited the conversion of LC3B-I to LC3B-II. (C) At $24 \mathrm{~h}$ after ionizing radiation of $6 \mathrm{~Gy}$, Eca-109/GFP-LC3 cells exhibited more punctate green fluorescent staining, consistent with autophagy, which decreased after combined therapy with ionizing radiation of $6 \mathrm{~Gy}$ and 3-MA. Magnification x40; scale bar, $200 \mu \mathrm{m}$. (D) Combined therapy with ionizing radiation of $6 \mathrm{~Gy}$ and 3-MA reduced the percent of cells with over 10 punctate green fluorescent dots $(\mathrm{P}<0.01)$.

Staining quantification. Images were imported into ImageJ and either converted to binomy with the quantification of the percent of the area positively stained or the determination of the relative intensity of the color staining of interest using the measure RGB feature. Data are expressed in arbitrary units per high powered field.

\section{Results}

Autophagy inhibitors disrupt autophagy in radiation-induced Eca-109 cells. 3-MA is typically used as an autophagy inhibitor. The extent of conversion from LC3B-I to LC3B-II reflects the level of autophagosome formation. Western blotting demonstrated that after radiation, the ratio of LC3B-II/LC3B-I was significantly increased in the Eca-109 cells, whereas the levels of LC3B-II/LC3B-I were decreased following 3-MA treatment in a dose-dependent manner (Fig. 1A and B). These results indicated that autophagy inhibitors disrupted the radiation-induced Eca-109 cell autophagy. Similar effects were noted in the Eca-109 cells transduced to express a GFP-LC3 fusion protein, in which radiation increased autophagy, as determined by the number of Eca-109 cells with punctuate green staining, the marker reduced by 3-MA. After radiation exposure, 3-MA decreased the number of cells with punctate green staining $(\mathrm{P}<0.01$; Fig. $1 \mathrm{C}$ and $\mathrm{D})$.

Inhibition of radiation-induced autophagy increases cell cycle arrest and cell death. Flow cytometry showed that when Eca-109 cells were treated with 3-MA, irradiation or combined therapy, the cell cycle distribution was different. When Eca-109 
A

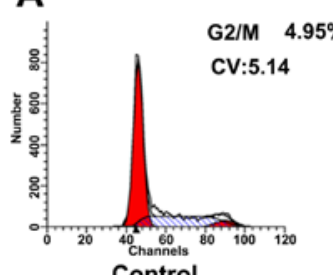
Control

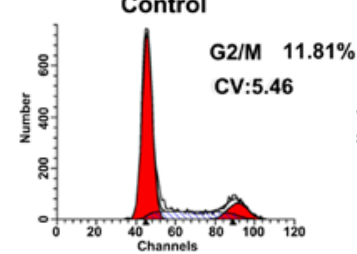

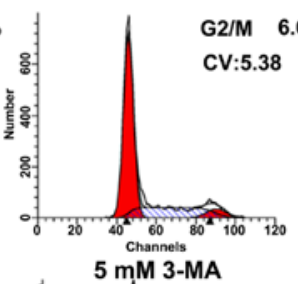

G2/M $6.64 \%$
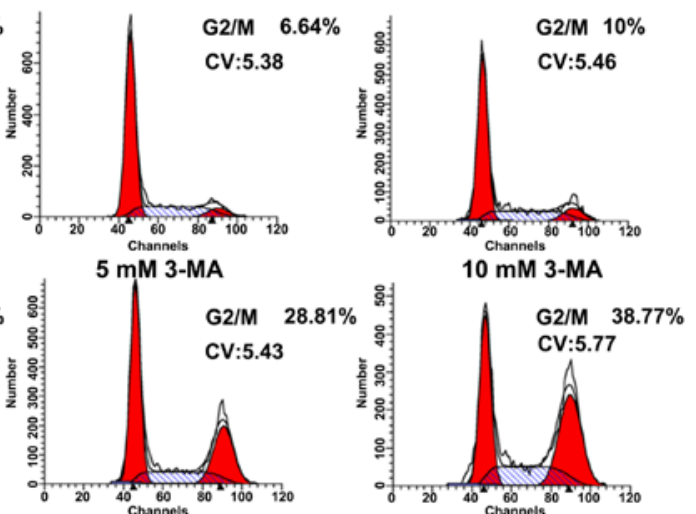

B

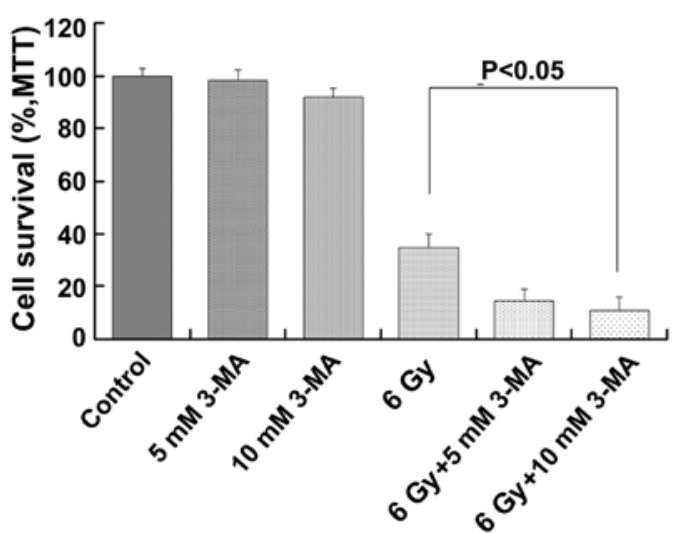

Figure 2. Inhibition of radiation-induced autophagy increases cell cycle arrest and cell death. (A) Detection of cell cycle phases by flow cytometry. (B) Eca-109 cells exhibited significantly decreased cell numbers after ionizing radiation of 6 Gy plus $10 \mathrm{mM} 3-\mathrm{MA}(\mathrm{P}<0.05)$, as assessed by the absorbance at $490 \mathrm{~nm}$ (reflecting number of cells) minus the background measured in the MTT assay.
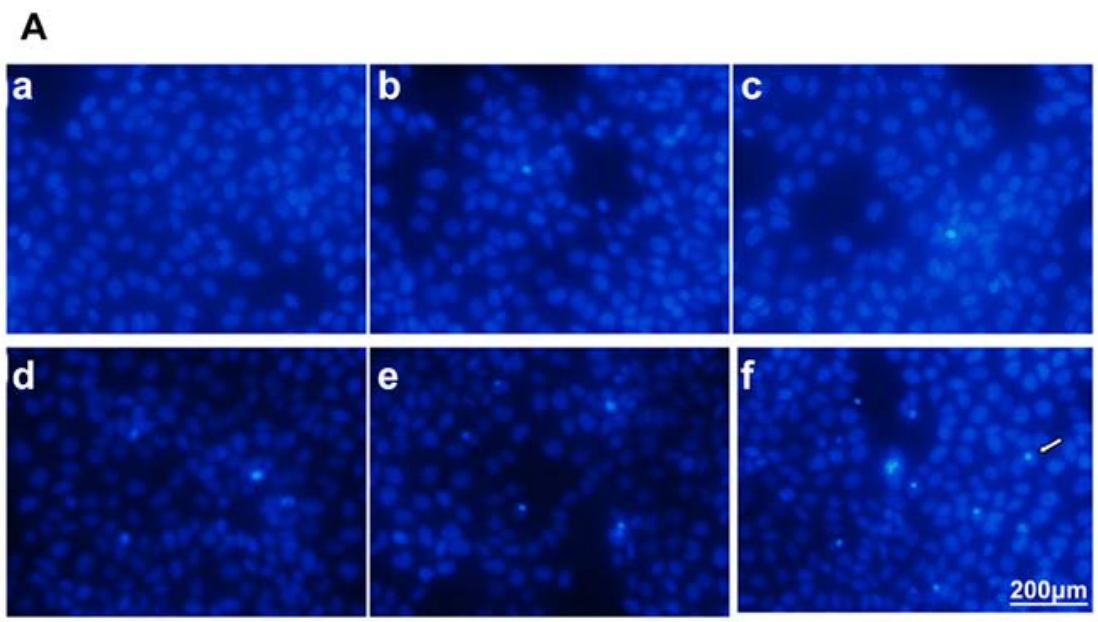

B
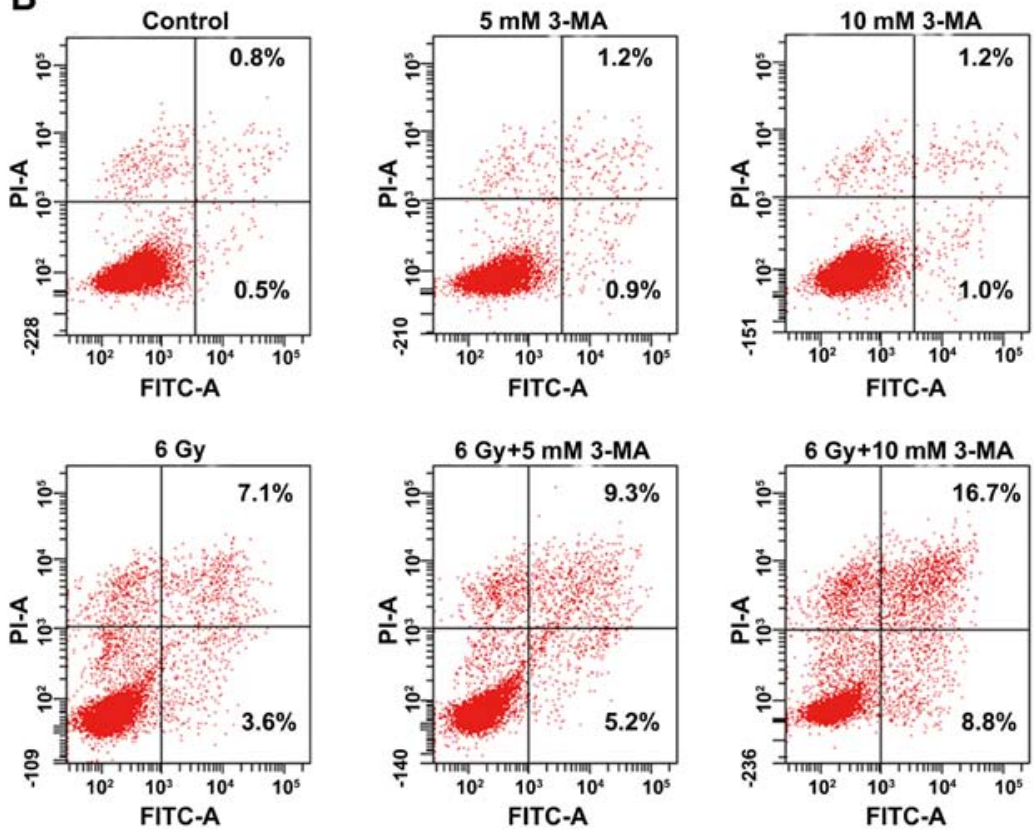

Figure 3. Inhibition of radiation-induced autophagy increases cell apoptosis. (A) Nuclear morphology changes were analyzed by fluorescence microscopy using Hoechst 33342 nuclear fluorescent dye staining. Magnification x40; scale bar, $20 \mu \mathrm{m}$. (B) Induction of apoptosis was evaluated by staining cells with Annexin V/PI. The dual parameter dot plots shows cells at early apoptosis in the lower-right quadrant (Annexin $\left.\mathrm{V}^{+} / \mathrm{PI}^{-}\right)$and cells at late apoptosis in the upper right quadrant (Annexin $\left.\mathrm{V}^{+} / \mathrm{PI}^{+}\right)$. 


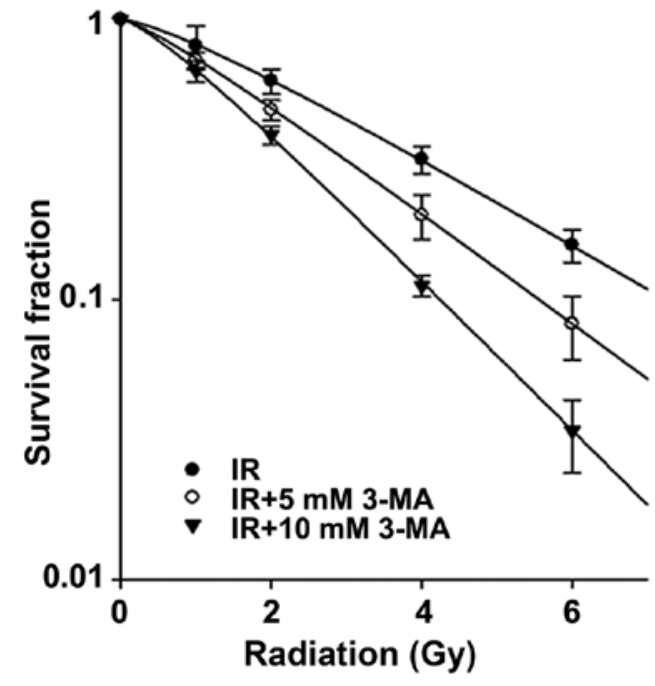

Figure 4. Clonogenic survival of Eca-109 cells after inhibition of radiation-induced autophagy. A clonogenic survival assay was performed to evaluate long-term survival. Cell survival curve of the Eca-109 cells treated with radiation alone or in combination with 3-MA (5 or $10 \mathrm{mM})$, as assessed using the linear-quadratic formula.

cells were treated with irradiation and 3-MA, we observed G2/M phase arrest of the Eca-109 cells; however, the Eca-109 cells in the G2/M phase were increased only briefly. Notably, the Eca-109 cells in the G2/M phase were significantly increased when the Eca-109 cells were treated with combined therapy for $24 \mathrm{~h}$ (Fig. 2A). We measured the cell survival after radiation exposure combined with 3-MA. 3-MA significantly decreased the number of viable Eca-109 cells after radiation exposure (Fig. 2B). Based on the survival-promoting effect of radiation-induced autophagy, we characterized the type of cell death occurring after radiation exposure when autophagy was inhibited. We stained Eca-109 cells with Hoechst 33342, a fluorescent dye that accumulates in apoptotic cells, and showed that the dye increased after the Eca-109 cells were treated with irradiation, 3-MA or a combination of both. In particular, Hoechst 33342 clearly accumulated following the combined therapy with irradiation and 3-MA. The percentages of early and late apoptotic cells were significantly increased after combined therapy with irradiation and 3-MA (Fig. 3).

Clonogenic survival of Eca-109 cells after inhibition of radiation-induced autophagy. To determine whether inhibition of autophagy sensitizes Eca-109 cells to radiation, we performed colony forming assays. As shown in Fig. 4, the number of surviving Eca-109 cells was significantly decreased with increasing concentrations of 3-MA. The SF2 (survival fraction at $2 \mathrm{~Gy}$ ) and D0 values (dose of radiation producing a $37 \%$ survival rate) were $61 \pm 5 \%$ and $2.75 \pm 0.26$ Gy when irradiation was used alone. However, for the combined therapy with irradiation and 3-MA, the SF2 and D0 values were decreased in a concentration-dependent manner and were $48 \pm 4 \%$ and $2.19 \pm 0.18$ Gy and $38 \pm 5 \%$ and $1.6 \pm 0.05$ Gy for 5 and $10 \mathrm{mM}$ 3-MA, respectively.

Dependence of radiation-induced autophagy on LKB1. Sapkota et al observed radiation-induced phosphorylation of
A

Control $2 \mathrm{~Gy} 4 \mathrm{~Gy} 6 \mathrm{~Gy}$
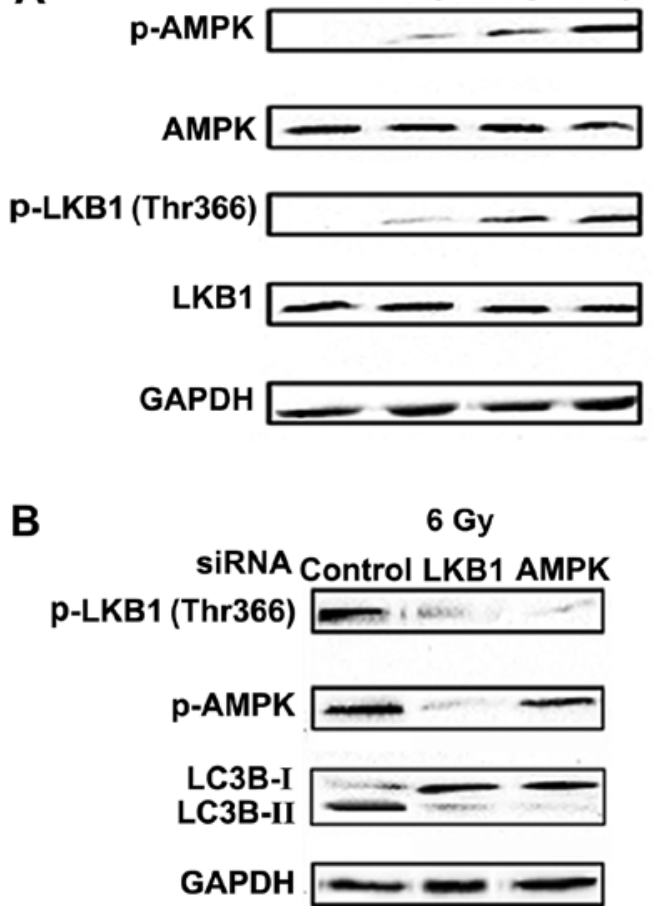

Figure 5. Radiation upregulates LKB1 and AMPK phosphorylation, contributing to aspects of radiation-induced autophagy. (A) Eca-109 cells after radiation exposure exhibited dose-dependent activation of LKB1 and AMPK (LKB1 and AMPK phosphorylation). (B) siRNA-mediated knockdown of LKB1 and AMPK in Eca-109 cells after ionizing radiation of 6 Gy exhibited reduced LC3-I to LC3-II conversion, and LKB1 siRNA reduced LKB1 and AMPK phosphorylation.

LKB1 at Thr-366 (8). After radiation exposure, both LKB1 and AMPK were activated in the Eca-109 cells. In particular, the expression of p-LKB1 (Thr-366) and p-AMPK was increased in a dose-dependent manner (Fig. 5A). Furthermore, siRNA-mediated knockdown of LKB1 or AMPK in the Eca-109 cells blocked the radiation-mediated LC3B-I to LC3B-II conversion, and LKB1 siRNA reduced LKB1 and AMPK phosphorylation (Fig. 5B). We then investigated the transcriptional changes to radiation-induced autophagy. The changes in LC3B transcripts in the negative control relative to the LKB1 siRNA after radiation exposure were significant $(\mathrm{P}<0.05$; Fig. 6$)$.

Combined inhibition of autophagy and ionizing radiation exerts a potent antitumor effect in vivo. Chloroquine (CQ) is used to inhibit autophagy in vivo, partly because it is the only FDA-approved autophagy inhibitor. We then determined whether CQ counteracted the survival-promoting effects of radiation-induced autophagy by treating subcutaneous tumors derived from Eca-109 cells with the autophagy inhibitor CQ and/or ionizing radiation of $6 \mathrm{~Gy}$. After 27 days, the tumor volumes differed between the 4 treatment groups $(\mathrm{P}<0.05)$, and combined therapy (CQ+6 Gy) inhibited tumor growth significantly vs. ionizing radiation of $6 \mathrm{~Gy}$ alone $(\mathrm{P}<0.05)$ (Fig. 7A). Furthermore, ionizing radiation of 6 Gy significantly increased LC3B expression and decreased p-LKB1 (Thr366) simultaneously. Compared to PBS or CQ treatment alone 


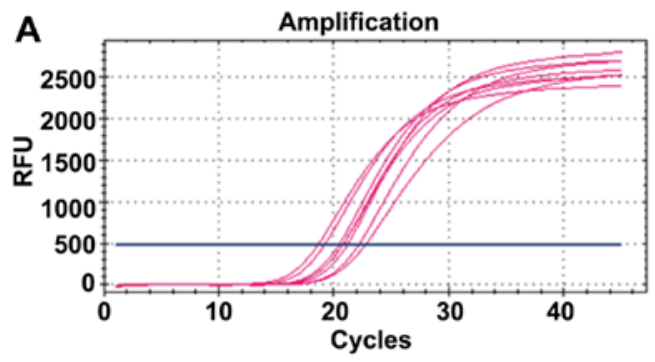

LC3B
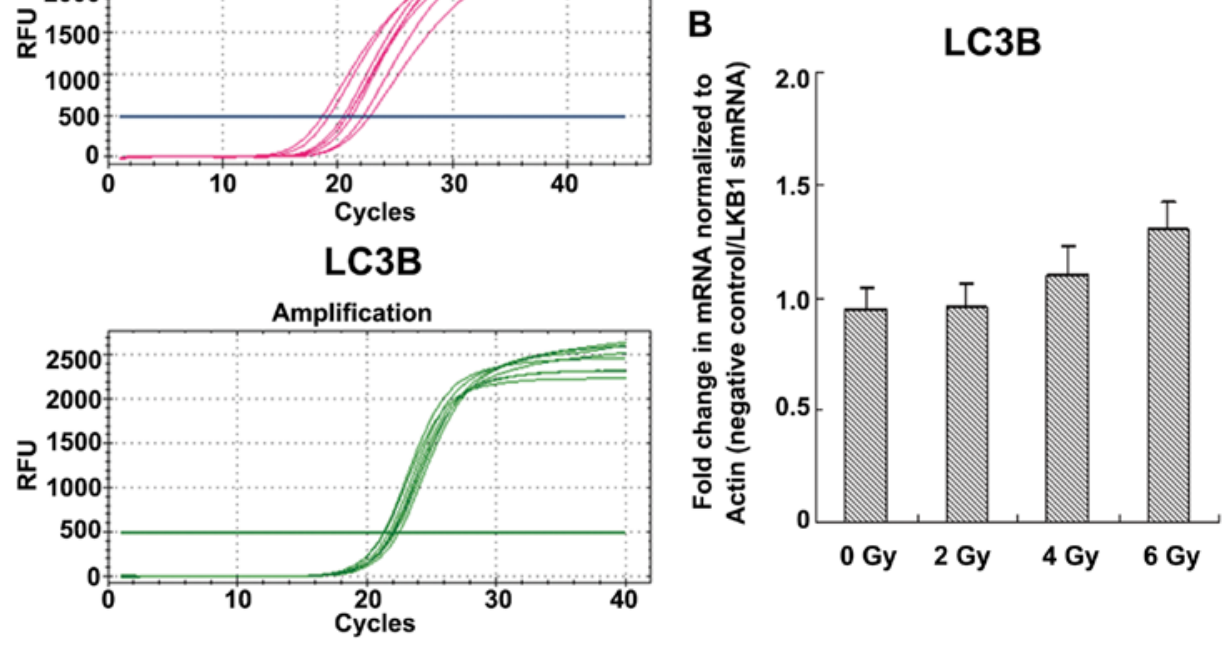

\section{ACTIN}

Figure 6. Real-time RT-PCR to determine autophagy-related transcripts in the negative control relative to LKB1 siRNA. (A) Real-time RT-PCR analysis was used to detect LC3 expression in Eca-109 cells in the negative control relative to LKB1 siRNA after radiation exposure. (B) Fold change in the levels of LC3 after normalization relative to actin in the negative control relative to LKB1 siRNA after radiation exposure. Error bars represent standard deviations.
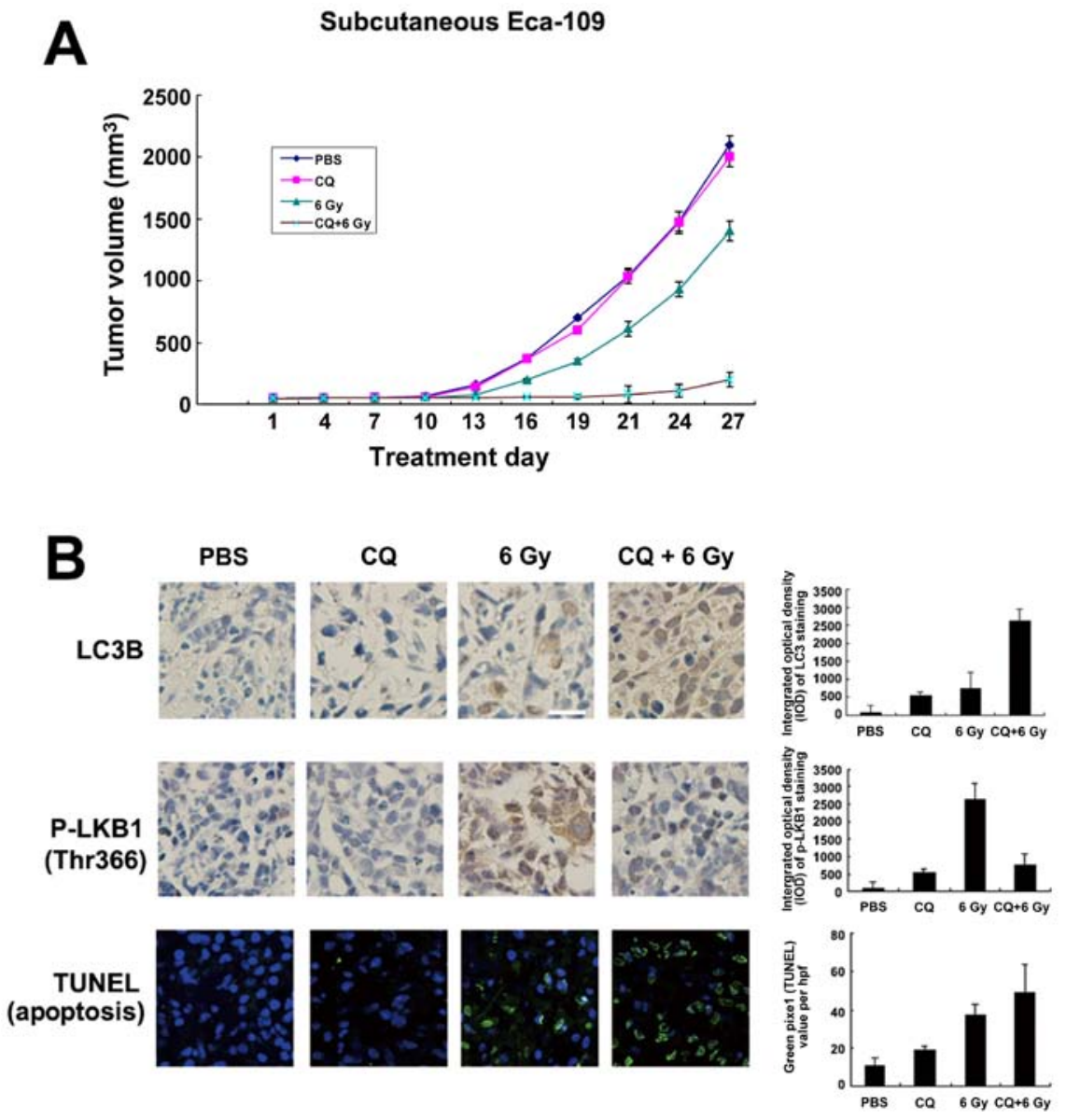

Figure 7. Autophagy inhibitor chloroquine combined with ionizing radiation inhibits Eca-109 tumor growth in vivo. (A) Subcutaneous tumors in athymic mice were treated with PBS, chloroquine (CQ), ionizing radiation of $6 \mathrm{~Gy}$, and CQ+6 Gy. After 27 days, there were significantly different tumor volumes among the groups $(\mathrm{P}<0.05)$. Combined therapy with ionizing radiation of $6 \mathrm{~Gy}$ and $\mathrm{CQ}$ inhibited tumor growth in a prolonged and statistically significant manner relative to either agent alone ( $\mathrm{P}<0.01$, ionizing radiation of $6 \mathrm{~Gy}$ vs. $\mathrm{CQ}+6 \mathrm{~Gy} ; \mathrm{P}<0.01, \mathrm{CQ}$ vs. $\mathrm{CQ}+6 \mathrm{~Gy})$. (B) Integrated optical density (IOD) quantification of LC3B expression increased in the $\mathrm{CQ}+6 \mathrm{~Gy}$ group $(\mathrm{P}<0.05)$. Integrated optical density (IOD) quantification of $\mathrm{p}-\mathrm{LKB} 1$ (Thr366) expression decreased in the $\mathrm{CQ}+6$ Gy group $(\mathrm{P}<0.05)$. TUNEL staining was increased in the $\mathrm{CQ}+6 \mathrm{~Gy}$ groups compared to the 6 Gy groups $(\mathrm{P}<0.05)$. DAPI nuclear counterstaining is blue. Magnification $\mathrm{x} 40$; scale bar, $100 \mu \mathrm{m}$. 
$(\mathrm{P}<0.05)$, combined therapy $(\mathrm{CQ}+6 \mathrm{~Gy})$ increased LC3B expression and decreased p-LKB1 (Thr366) ( $\mathrm{P}<0.05$; Fig. 7B). Cell death in these xenografts was characterized using TUNEL staining to detect cells in late apoptosis. Staining was increased in the CQ+6 Gy groups compared to the $6 \mathrm{~Gy}$ groups $(\mathrm{P}<0.05$; Fig. 7B).

\section{Discussion}

Autophagy is a fundamental and phylogenetically conserved self-degradation process that is characterized by the formation of double-layered vesicles (autophagosomes) around intracellular cargo for delivery to lysosomes and proteolytic degradation (10). Autophagy has two functions in cancer. It can be tumor suppressive through the elimination of oncogenic protein substrates, toxic unfolded proteins and damaged organelles or, alternatively, it can be tumor promoting in established cancers via autophagy-mediated intracellular recycling that provides substrates for metabolism and maintains the functional pool of mitochondria (11). Autophagy, an alternative form of programmed cell death, contributes significantly to the anti-neoplastic effects of radiation therapy.

The ability of autophagy to capture, degrade, eliminate and recycle intracellular components affects metabolism, enables host defenses, remodels the proteome, regulates trafficking, alters signaling and influences cellular interactions (10). In this study, we demonstrate that Eca-109 cells treated with autophagy inhibitors after radiation exhibited decreased survival and upregulated apoptosis. Our in vivo data showed increased TUNEL staining in CQ plus radiation-treated xenografts, suggesting an increased number of apoptotic cells during combined treatment. We also found that the combined treatment induced G2/M cell cycle arrest, which may contribute to cell death. In addition, we also demonstrated that 3-MA, an inhibitor of autophagy, significantly decreased the clonogenic survival of the Eca-109 cells. These findings indicate that autophagy functions as a tumor-protective effect after exposure to stressors, such as radiation. A few similar reports have demonstrated that cell apoptosis can delay or evade death via the autophagosome-lysosome pathway in response to various stressors (12).

There is ample evidence that radiation-induced cell death is affected by various related biochemical processes in the autophagic pathways. Moretti et al reported that radiation may activate autophagy via the adaptive endoplasmic reticulum stress response (13). Nam et al reported the activation of autophagy via inhibition of the mTOR pathway, leading radioresistant cancer cells to senescence (6). We found that LKB1 was phosphorylated at Thr-366 after radiation exposure in a dose-dependent manner, and AMPK was also activated. Both LKB1 and AMPK could contribute to autophagy, with P27(kip1) activation as a possible mechanism (14). These findings suggest an internal relationship between autophagy, LKB1, AMPK and radiation. We found that radiation-mediated LC3B-I to LC3B-II conversion and overall LC3B degradation was dependent upon both LKB1 and AMPK.
Interestingly, CQ decreased p-LKB1 expression in radiotherapy xenografts. CQ is a late autophagy inhibitor, acting on cells downstream of LKB1 (15). Theoretically, CQ would not affect P-LKB1 expression based on the regulatory mechanism of autophagy. The differences may reflect a potential negative feedback system of autophagy when autophagosomes rapidly increase in vivo. Future studies are necessary to clarify this contradiction.

In conclusion, our findings reveal an important relationship between radiation and autophagy signaling via LKB1 and show that autophagy promotes esophageal squamous carcinoma cell survival through pharmacological or genetic means. Targeting autophagy is a novel method to enhance the effect of radiation therapy as an anticancer modality.

\section{Acknowledgements}

This study was supported by Grant WX13B02 from the Foundation of Health and Family Planning Commission of Wuhan Municipality, China.

\section{References}

1. Enzinger PC and Mayer RJ: Esophageal cancer. N Engl J Med 349: 2241-2252, 2003.

2. Jemal A, Bray F, Center MM, Ferlay J, Ward E and Forman D: Global cancer statistics. CA Cancer J Clin 61: 69-90, 2011.

3. Sudo T, Iwaya T, Nishida N, Sawada G, Takahashi Y, Ishibashi M, Shibata K, Fujita H, Shirouzu K, Mori M, et al: Expression of mesenchymal markers vimentin and fibronectin: The clinical significance in esophageal squamous cell carcinoma. Ann Surg Oncol 20 (Suppl 3): S324-S335, 2013.

4. Siegel R, Naishadham D and Jemal A: Cancer statistics, 2012. CA Cancer J Clin 62: 10-29, 2012.

5. Graf D, Vallböhmer D, Knoefel WT, Budach W and Häussinger D: Multimodal treatment of esophageal carcinoma. Dtsch Med Wochenschr 139: 2141-2147, 2014 (In German).

6. Nam HY, Han MW, Chang HW, Kim SY and Kim SW: Prolonged autophagy by mTOR inhibitor leads radioresistant cancer cells into senescence. Autophagy 9: 1631-1632, 2013.

7. Zeng $X$ and Kinsella TJ: Impact of autophagy on chemotherapy and radiotherapy mediated tumor cytotoxicity: 'To live or not to live'. Front Oncol 1: 30, 2011.

8. Sapkota GP, Deak M, Kieloch A, Morrice N, Goodarzi AA, Smythe C, Shiloh Y, Lees-Miller SP and Alessi DR: Ionizing radiation induces ataxia telangiectasia mutated kinase (ATM)-mediated phosphorylation of LKB1/STK11 at Thr-366. Biochem J 368: 507-516, 2002.

9. Wu WK, Coffelt SB, Cho CH, Wang XJ, Lee CW, Chan FK, Yu J and Sung JJ: The autophagic paradox in cancer therapy. Oncogene 31: 939-953, 2012.

10. Barth S, Glick D and Macleod KF: Autophagy: Assays and artifacts. J Pathol 221: 117-124, 2010.

11. White E: Deconvoluting the context-dependent role for autophagy in cancer. Nat Rev Cancer 12: 401-410, 2012.

12. Carew JS, Kelly KR and Nawrocki ST: Autophagy as a target for cancer therapy: New developments. Cancer Manag Res 4: 357-365, 2012.

13. Moretti L, Cha YI, Niermann KJ and Lu B: Switch between apoptosis and autophagy: Radiation-induced endoplasmic reticulum stress? Cell Cycle 6: 793-798, 2007.

14. Sanli T, Steinberg GR, Singh G and Tsakiridis T: AMP-activated protein kinase (AMPK) beyond metabolism: A novel genomic stress sensor participating in the DNA damage response pathway. Cancer Biol Ther 15: 156-169, 2014.

15. He C and Klionsky DJ: Regulation mechanisms and signaling pathways of autophagy. Annu Rev Genet 43: 67-93, 2009. 